\title{
Vascular endothelial growth factor encoded by Parapoxviruses can regulate metabolism and survival of triple negative breast cancer cells
}

\author{
Dipayan Bose (1)', Sagarika Banerjee', Rajnish Kumar Singh'1, Lyn M. Wise ${ }^{2}$ and Erle S. Robertson (1)
}

\begin{abstract}
Dysbiotic microbiomes are linked to many pathological outcomes including different metabolic disorders like diabetes, atherosclerosis and even cancer. Breast cancer is the second leading cause of cancer associated death in women, and triple negative breast cancer (TNBC) is the most aggressive type with major challenges for intervention. Previous reports suggested that Parapoxvirus signatures are one of the predominant dysbiotic viral signatures in TNBC. These viruses encode several genes that are homologs of human genes. In this study, we show that the VEGF homolog encoded by Parapoxviruses, can induce cell proliferation, and alter metabolism of breast cancer and normal breast cells, through alteration of MAPK-ERK and PI3K-AKT signaling. In addition, the activity of the transcription factor FoxO1 was altered by viral-encoded VEGF through activation of the PI3K-AKT pathway, leading to reprogramming of cellular metabolic gene expression. Therefore, this study provides new insights into the function of viral-encoded VEGFs, which promoted the growth of the breast cancer cells and imparted proliferative phenotype with altered metabolism in normal breast cells.
\end{abstract}

\section{Introduction}

Breast cancer is one of the most frequent cancers affecting 1 in 8 women in the United States ${ }^{1}$. There are four major types of human breast cancers, and among them triple negative breast cancer (TNBC) is the most aggressive form with the poorest prognosis ${ }^{2}$. Over the last few decades many studies to develop a better understanding of the molecular pathways have been explored so that potential targets can be identified for the development of new therapeutics. The development of breast cancer, similar to other cancer types is also linked to lifestyle, ethnicity, genetic background, and obesity. However, recent studies have identified microbiome present in the tumor microenvironment as another potential

\footnotetext{
Correspondence: Erle S. Robertson (erle@pennmedicine.upenn.edu) ${ }^{1}$ Department of Otorhinolaryngology-Head and Neck Surgery, Perelman School of Medicine, Tumor Virology Program, Abramson Cancer Center, University of Pennsylvania, Philadelphia, PA 19104, USA

${ }^{2}$ Department of Pharmacology and Toxicology, School of Biomedical Sciences, University of Otago, Dunedin, New Zealand
}

Edited by N. Robinson contributor which plays a major role in cancer development $^{3,4}$. It is also well-established that greater than $18 \%$ of human cancers are tightly linked to the presence of infectious microorganisms, which were demonstrated to play crucial roles in their pathogenesis. Chronic infections are also identified as contributors which have been linked to ongoing inflammatory responses ${ }^{5,6}$. It should also be noted that different microbial antigens and secretory factors can also play critical roles in driving cancer pathogenesis ${ }^{7}$.

The human body is colonized by microorganisms estimated to be equivalent in numbers to that of human cells. These are predominantly either commensals or mutualistic microorganisms ${ }^{8}$. An imbalance in this microbial population results from colonization of pathogenic microorganisms, which leads to the establishment of a dysbiotic microbiome and initiation of different pathogenic activities. This dysregulation in the microbiome is also now accepted as one of the major drivers in cancer pathogenesis ${ }^{9,10}$.

\section{(c) The Author(s) 2020}

(c) (i) Open Access This article is licensed under a Creative Commons Attribution 4.0 International License, which permits use, sharing, adaptation, distribution and reproduction cc) in any medium or format, as long as you give appropriate credit to the original author(s) and the source, provide a link to the Creative Commons license, and indicate if changes were made. The images or other third party material in this article are included in the article's Creative Commons license, unless indicated otherwise in a credit line to the material. If material is not included in the article's Creative Commons license and your intended use is not permitted by statutory regulation or exceeds the permitted use, you will need to obtain permission directly from the copyright holder. To view a copy of this license, visit http://creativecommons.org/licenses/by/4.0/. 
Previously, we reported that TNBC samples are characterized by the presence of an array of different microbial agents, which included Arcanobacterium, Brevundimonas, Sphingobacteria, Providencia, Prevotella, Brucella, Escherichia, Actinomyces bacteria; viruses which included Herpes, Parapox, Flavivirus, Polyoma and Retrovirus families; Fungi namely Piedra, Foncecaea, Phialophora, and Paecilomyces. This study was performed on a cohort of 100 TNBC samples 17 matched controls and 20 nonmatched controls ${ }^{11}$. Analyses of these tumor samples identified Parapoxvirus signatures as one of the most abundantly found viral signatures present ${ }^{11}$. These viral agents have been previously reported to encode homologs of human VEGF ${ }^{12,13}$. Three sub-groups of Parapoxviruses (ORFV, PCPV, and BPSV) encodes viral homologs of human VEGFs (VEGF-E), which bears significant polypeptide sequence homology with the human cellular VEGF-A $(27 \%, 29 \text {, and } 46 \% \text { respectively })^{14}$. Human VEGF-A is produced by different kinds of cells including endothelial, hematopoietic, and stromal cells in response to hypoxia and has binding potential with three different receptors namely VEGFR1, 2 and $3^{15}$ whereas the viral VEGF-E is only reported to bind with human VEGFR2 and neuropilin1 $1^{16}$, to initiate downstream cellular processes.

Cancer cells are characterized by their metabolic reprogramming which is required to provide the necessary energy requirement for its growth and survival. Instead of undergoing oxidative phosphorylation, they rapidly metabolize glucose in an anaerobic manner to produce lactic acid by the phenomenon known as the Warburg effect ${ }^{17}$. Cancer cells metabolize glucose at a faster rate by upregulating glycolysis and downregulating glucose assimilation and gluconeogenesis ${ }^{18}$. Similarly, the fatty acid and amino acid biosynthetic pathways are also dysregulated. Notably, the glutamine metabolism and fatty acid oxidation pathways are the most dramatically affected pathways in breast cancer ${ }^{19,20}$. There are numerous previous reports about small molecules which targets against arginase (ARG) and nitric oxide synthase $(\mathrm{NOS})^{21}$, arginine-succinate lyase $(\mathrm{ASL})^{22}$, and the branched-chain amino acid amino transferase (BCAT1) ${ }^{23}$ of the amino acid metabolism which are all expressed in higher concentrations in breast cancer. Fatty acid metabolic genes like fatty acid synthase $(\mathrm{FAS})^{24}$, carnitine palmitoyl transferase I (CPT1) ${ }^{25}$, acetyl-CoA carboxylase$\alpha(\mathrm{ACC})^{26}, 3$-hydroxy-3-methylglutaryl-CoA synthase $(\text { HMGCS })^{27}$, and acyl-CoA cholesterol acyl transferase $(\mathrm{ACAT})^{28}$, are also highly expressed in different cancers. However, it is well documented that glycolysis and the Kreb's cycle, not only controls the flow of carbon in carbohydrate metabolism, but can indirectly affect lipid and amino acid metabolism by modulating the availability of the internal carbon pool of the cell ${ }^{19,29}$.
TNBC is one of the deadliest types of breast cancer because of its aggressiveness and the lack of responsiveness to different lines of available treatments. TNBC are negative to human epidermal growth factor receptor 2 (HER), progesterone receptor (PR), and estrogen receptors (ER), but are typically positive for receptor tyrosine kinase (RTK) receptors ${ }^{30,31}$. Previous studies have suggested that epidermal growth factor (EGF) binds to its cognate receptor, epidermal growth factor receptor (EGFR) to initiate downstream signaling that leads to the synthesis of cellular VEGF, which, in turn, binds to VEGFR and promotes cell proliferation ${ }^{32}$. Besides angiogenesis and cell proliferation, VEGF is also linked to maintenance of cell survival through regulation of metabolism and autophagy ${ }^{33}$. Furthermore, the PI3K-AKT and ERK-MAPK are the two central pathways that are involved in modulating transcription of metabolic genes $^{34,35}$, and, therefore, contribute to the regulation of cellular metabolism.

In this study, we have identified Parapoxvirus DNA elements as a potential signature for a dysbiotic microbiome, and the viral VEGF-E is one of the factors modulating altered metabolism and cellular proliferation in TNBC through activation of the PI3K-AKT and ERKMAPK pathway. Moreover, we have demonstrated that VEGF-E can induce higher proliferation and increased metabolism in normal breast cells. Our findings strongly suggest that the VEGF-E encoded by these Parapoxviruses can also reprogram normal breast cells to a hyperproliferative state with altered metabolism similar to that of cancer cells. Our study provides further evidence of dysbiotic microbiomes and their encoded secretory antigens capable of reprogramming the tumor and the surrounding cells, and thus contributing to progression of disease.

\section{Materials and methods \\ Human study samples}

This study was performed on a cohort of 11 TNBC samples (EXTN) and their corresponding surrounding non-cancerous tissues or matched control (EXMC), and 10 tissue samples from healthy individuals as nonmatched control (NC). The NC samples are breast tissues from normal individuals. No patient identifiers were available to the laboratory staff, and their use were approved by the institute (IRB protocol number 832857). All experiments with formalin-fixed paraffin-embedded tissues samples (FFPE) were performed according to relevant guidelines and regulations, which were approved by the institutional committee of the Perelman School of Medicine, University of Pennsylvania. No information was obtained related to the treatment regimens of these breast cancer patients. All these tissues were obtained as deidentified archived samples. Tumor samples were received either as $10 \mu \mathrm{m}$ rolls or in $10 \mu \mathrm{m}$ sections on slides. 
Pathology staff reviewed the case histories, confirmed the tumor types and demarcated the cancer cells. Proper care was taken during dissection of the samples to control for environmental contamination and to minimize the potential cross contamination between samples by sterilizing the blades and the field ${ }^{36,37}$.

\section{PathoChip design}

The details of the PathoChip array have been described previously $^{11}$. It comprises 60,000 probes that represents over 6000 different microorganisms (viruses, bacteria, fungi, and parasites) listed in GenBank. The arrays were manufactured by Agilent Technologies Inc. and contains eight replicate arrays per slide. Probes are of 60 nucleotide DNA oligomers that targets genomic regions of viruses, prokaryotic and eukaryotic microorganisms. The PathoChip technology, combined with PCR and capture-nextgeneration sequencing (NGS), is a valuable strategy for detecting and identifying pathogens in human cancers and infection-related pathologies.

The DNA and RNA were extracted from the samples and were subjected to whole-genome and transcriptome amplification (referred here as WTA) using the TransPlex Complete Whole Transcriptome Amplification Kit (Sigma-Aldrich, St. Louis, MO), using $50 \mathrm{ng}$ each of RNA and DNA as input. The WTA were processed as described earlier $^{11}$. The data were extracted from the Agilent SureScan G4900DA array scanner and were analyzed as described previously ${ }^{11,36,37}$. The $\mathrm{R}$ program was used to normalize the data and on the normalized signals $t$-test was applied to select probes significantly present in cancer samples by comparing cancer samples versus controls and to select probes significantly present in the breast cancer samples versus the controls.

PathoChip results were validated using specific primer pairs for the target microorganism. Quantitative PCR with cut-off of 35 cycles for each of amplicons were used for each of the primer pairs. Samples in which there is at least minimum amplification (above threshold values) are regarded as positive and all others were considered as negative.

The WTA prepared form the tissues samples were used as template for the real-time PCR when determining the expression of the selected metabolic genes. Primer sequences are provided in SI Tables 1-3.

\section{Cell lines, plasmids, antibodies, and inhibitors}

The TNBC cell line (MDA-MB-231), and human mammary epithelial cells (HMEC) were purchased from ATCC and were maintained in recommended culture condition as per the ATCC instructions ${ }^{38}$. The viral VEGF-E homologs (PCPV-VEGF, ORFV-VEGF, and BPSV-VEGF) were a kind gift from Dr. L.M. Wise, University of Otago, Dunedin New Zealand ${ }^{39}$. Antibodies against GFP, PCNA, GAPDH, p-Erk, p-MEK, p-Raf, AKT, FoxO1, H2A were purchased from Santa Cruz Biotechnology Inc Dallas, TX, and ERK, PI3k110 $\alpha$, and pAKT was purchased from Cell Signaling Technologies Inc. Danvers, MA and anti-BrdU antibody from Invitrogen, Thermo Fisher (SI Table 4). The secondary antibodies for western blot are IR conjugated and were from LI-COR Inc. Lincoln, NE, while for microscopy the secondary antibody is Alexa 594 conjugated purchased from Invitrogen Inc. Carlsbad, CA.

LY294002, an inhibitor for PI3K was purchased from Sigma Aldrich Inc. St. Louis, MO. MDA-MB-231 cells were treated with $50 \mu \mathrm{M}$ and HMEC cells with $30 \mu \mathrm{M}$ of LY294002 and incubated for $24 \mathrm{~h}$ before being used for assays.

\section{Lentiviral transduction and preparation of stable cell line}

The pLVX-AcGFP Lentiviral plasmid was double digested with AccI and BamH1 and was used to clone the three viral VEGF-E homologs with a signal sequence fused with GFP at the C-terminal. For MDA-MB-231 and HMEC cells with VEGF-E stable expression, the VEGFexpressing plasmid pLVX-AcGFP-VEGF or vector alone, was individually co-transfected with Lentivirus packageexpressing plasmids (Rev, vesicular stomatitis virus G [VSVG], and glycoprotein) into HEK293T cells to generate virus. The packaged viruses were used to individually transduce MDA-MB-231 and HMEC cells, with selection by $1.5 \mu \mathrm{g} / \mathrm{ml}$ puromycin for MDA-MB-231 and $1 \mu \mathrm{g} / \mathrm{ml}$ for HMEC cells until all the cells are positive to GFP . The expression of the GFP fused VEGF-E proteins were tested by western blot using antibody against GFP and immunofluorescence analysis for GFP signals.

\section{Western blot and confocal microscopy}

Cell lysates were prepared from $10^{6}$ cells, and total proteins were isolated. $40 \mu \mathrm{g}$ of proteins were loaded in each well and were resolved on SDS-polyacrylamide gels followed by wet transfer to nitrocellulose membrane. 5\% skimmed milk/ BSA was used for blocking at room temperature for $1 \mathrm{~h}$ with gentle shaking. The membranes were incubated on primary antibodies for overnight at $4{ }^{\circ} \mathrm{C}$ followed by $30 \mathrm{~min}$ washing with TBST and probing with infrared (IR) dye conjugated secondary antibody. Membranes were scanned using an Odyssey scanner (LICOR Inc., Lincoln, NE) for detection of specific antigens and the band intensities were measured using the ImageQuant, Odyssey software. The band intensities were normalized with that of the loading control (GAPDH) and the fold intensity values were represented below each blot.

For confocal microscopy, cells were plated over coverslips. Coverslips were washed with ice cold PBS and fixed with $4 \%$ paraformaldehyde for 30 mins at room temperature and perforated by $0.05 \%$ of TritonX-100 for 
15 mins. Perforated cells were washed with PBS and incubated with primary antibody $(1: 150)$ overnight at $4{ }^{\circ} \mathrm{C}$, followed by 30 min washing with PBST and probing with Alexa fluorophore-conjugated secondary antibody $(1: 300)$ for $1 \mathrm{~h}$ before being washed with PBST, stained with DAPI and fixed on glass slides. The images were observed using an Olympus F300 Confocal Microscope. Microscopic images were analyzed using ImageJ (Adobe Inc., San Jose, $\mathrm{CA}$ ), and the co-localization co-efficient (Pearson's coefficient, r) was measured by the Coloc2 program of ImageJ, and the values were tabulated in graphical format. Each bar represents the value of at least 10 microscopic fields each from three independent experiments.

\section{Colony formation and cell proliferation assay}

$10^{5}$ transfected or non-transfected cells were plated with DMEM supplemented with 5\% BGS in $10 \mathrm{~cm}$ dish and after 5 days cells were fixed with $4 \%$ paraformaldehyde, and stained with $0.05 \%$ of crystal violet solution overnight, and visualized using a BioRad Chemidoc Imaging system (BioRad Inc. Hercules, CA). The colony number and size were quantitated using ImageJ.

For cell proliferation assays, $10^{6}$ cells were washed with PBS and treated with $10 \mu \mathrm{M}$ BrdU in complete growth medium and incubated for $24 \mathrm{~h}$ at $37^{\circ} \mathrm{C}$ in a $\mathrm{CO}_{2}$ incubator. Cells were washed twice with PBS and fixed with $4 \%$ PFA and permeabilized with $0.05 \%$ of TritonX- 100 for $15 \mathrm{~min}$ and washed with PBS before being stained with anti-BrdU antibodies conjugated with PE fluorophore ${ }^{40}$ and the fluorescence was checked with a flow cytometer (Becton-Dickinson Inc., San Jose, CA) and the data were analyzed by FlowJo Software (Treestar, Inc., San Carlos, CA). The Mean fluorescence intensity (MFI) was calculated and results are represented in the provided graphs.

For MTT assay $10^{4}$ cells were plated in each well of 96 well plate and grown for overnight. The exhausted media is discarded and the cells are treated with $100 \mu \mathrm{l}$ of $5 \mathrm{mg} / \mathrm{ml}$ of MTT solution prepared in serum free media for $4 \mathrm{~h}$ at $37^{\circ} \mathrm{C}$ in dark and $100 \mu \mathrm{l}$ of DMSO is added to solubilize and dissolve the formazan formed and the absorbance was checked at $590 \mathrm{~nm}$ in Cytation 5 (BioTek, VT, United States) and plotted in Graphpad Prism.

\section{Isolation of nuclear proteins}

Nuclear lysate was prepared according to the method previously described ${ }^{41} \cdot 10^{7}$ cells are washed with PBS, dislodged and pelleted by centrifugation and resuspended in the cell lysis buffer [10 mM HEPES; pH 7.5, $10 \mathrm{mM}$ $\mathrm{KCl}, 0.1 \mathrm{mM}$ EDTA, $1 \mathrm{mM}$ dithiothreitol (DTT), 0.5\% Nonidet-40 and $0.5 \mathrm{mM}$ PMSF along with the protease inhibitor cocktail (Sigma)] and allowed to swell on ice for 15-20 min with intermittent mixing. Tubes are vortexed to disrupt cell membranes and then centrifuged at $12,000 \times g$ at $4{ }^{\circ} \mathrm{C}$ for $10 \mathrm{~min}$.
The pelleted nuclei were washed 3 times with the cell lysis buffer and resuspended in the nuclear extraction buffer containing $20 \mathrm{mM}$ HEPES (pH 7.5), $400 \mathrm{mM} \mathrm{NaCl}$, $1 \mathrm{mM}$ EDTA, $1 \mathrm{mM}$ DTT, $1 \mathrm{mM}$ PMSF with protease inhibitor cocktail and incubated in ice for $30 \mathrm{~min}$. The nuclear extract was collected by centrifugation at $12,000 \times g$ for $15 \min$ at $4{ }^{\circ} \mathrm{C}$.

\section{RNA isolation, CDNA preparation, and real-time PCR}

RNA isolation was performed as per the standard method using TRizol reagent (Ambion, Grand Island, NY) and phenol-chloroform extraction. $2 \mu \mathrm{g}$ of total RNA was used to prepare the cDNA by a random priming method using the Superscript cDNA synthesis kit (Applied Biosystems Inc., Foster City, CA). The cDNA were diluted 10 times with $1 \mu \mathrm{l}$ of the diluted cDNA used for each $10 \mu \mathrm{l}$ volume of PCR reaction, with Power SYBR green PCR reagent (Applied Biosystems Inc., Carlsbad, CA) and Step One Plus, or Quant Studio PCR system (Applied Biosystems Inc., Carlsbad, CA). All the primers were designed from NCBI and were purchased from Integrated DNA Technologies Inc. (Coralville, IA). All real-time PCR assays were performed in duplicates, with at least two experimental repeats for each gene. The heat maps were generated by using the online Heatmapper software $^{42}$. The sequence of primers used for the PCR reactions are provided in SI Tables 1-3.

\section{Results}

Analysis of microbial population associated with triple negative breast cancer

Using Pathochip technology ${ }^{11,43}$ we screened 11 TNBC samples (EXTN) and their corresponding adjacent normal tissues as matched control (EXMC), and 10 healthy breast tissues samples as non-matched control (NC) for the presence of nucleic acids from a wide range of different microorganisms. Unique and common microbial signatures associated with the TNBC samples were compared to that of the matched and non-matched control and was tabulated in the form of a heat map (Fig. 1A). The probes which had a significantly higher hybridization signal ( $p$-value $<0.05, \log 2$ fold change in hybridization signal $>1$ ) were regarded as positive.

In this study, we analyzed the Pathochip data which identified 9 viruses, 7 bacteria, and 6 fungi with the highest hybridization signal and are most prevalent in the TNBC samples compared to the non-matched control. Among the 9 virus signatures identified to be unique in the tumor samples, Pox, Papilloma, and the Reo virus families were the most abundant with more than $90 \%$ prevalence among the tumor tissues, compared to $35-40 \%$ in matched control and less than $10 \%$ in non-matched control. Our Pathochip analyses showed that signatures for Poxviruses had the highest hybridization signals followed by Papilloma viruses (Fig. 1B). 


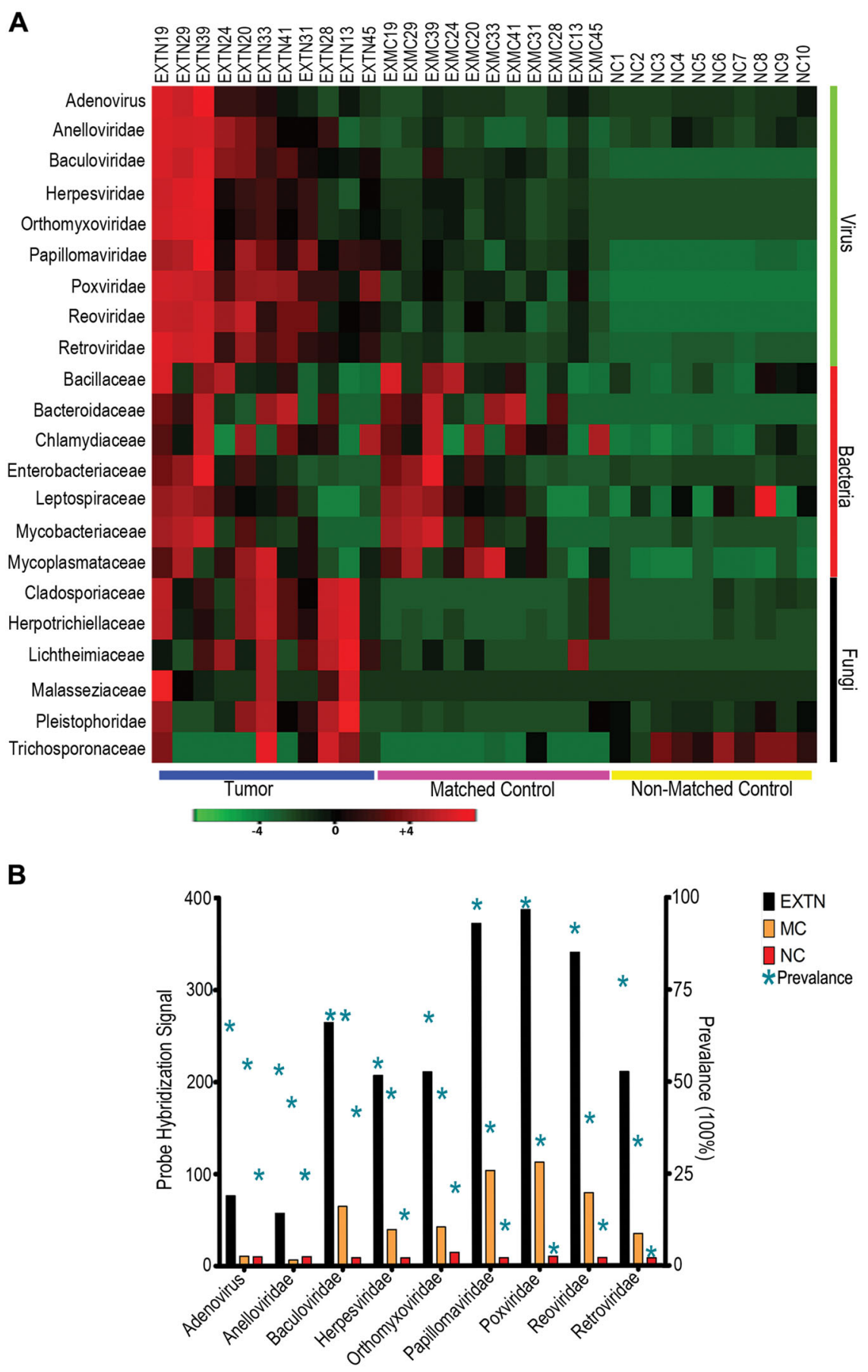

Fig. 1 Detection of viral, bacterial, and fungal signatures in the tumor samples (EXTN), its corresponding matched control (EXMC) and non-matched control (NC). A Signals for microbial signatures were detected in the EXTN, EXMC, and NC and are shown as heat maps of microorganism (y-axis) hybridized to the tumor samples and both matched (MC) and non-matched control (NC) samples ( $x$-axis). The matched controls were obtained from the adjacent normal breast tissues of the breast cancer samples. B Total hybridization signal for the most abundant viruses for all the tumor samples, matched control and the non- matched control were plotted. The prevalence of each of the virus in the different samples were represented with asterisk above the bar.

To validate the presence or absence of Poxvirus in the tissue samples, primers specific for the three different strains of Poxvirus (SI Table 1) were used and real-time
PCR was performed. DNA isolated from the strains of virus were used as positive control and DNA from MDAMB-231 cells were used as negative control. The results 
showed that only two tumor sample (EXTN28, 33) was negative for both ORFV and BPSV signatures while EXTN20, 41, 45 was negative for PCPV signatures (SI Fig. 1A). In matched controls 4,5 , and 7 samples among a total of 11 samples were found to be negative for signatures of ORFV, PCPV, and BPSV respectively (SI Fig. 1B). Among the non-matched control all samples were negative for Poxvirus genome signatures except NC3, which was found to be positive for BPSV genomic DNA (SI Fig. 1C).

\section{Detection of viral-encoded VEGF-E homolog in tissue samples}

The presence of VEGF-E, which represents homologs of human VEGFA encoded by the parapox virus ${ }^{39,44}$ in the tissue samples was confirmed by real-time PCR using primers specific for the three types of Poxviruses-encoded VEGF-E homologs, and human VEGFA was used as positive control. The real-time PCR analyses are represented as a heat map in (SI Fig. 2). Among the tumor samples, two samples (EXTN33 and EXTN28) were ORFV-VEGF-E negative, three samples (EXTN28, 39, 33) were BPSV-VEGF-E negative and four samples (EXTN20, 24, 45 and 41) were PCPV-VEGF negative. All other amplifications were positive for either ORFV, BPSV and PCPV (SI Fig. 2, left panel). Analysis of the matched controls showed that four samples (EXMC 28, 29, 31, and 33) were ORFV-VEGF-E negative, six samples (EXMC $13,20,39,45,33,31)$ were PCPV-VEGF-E negative, and six samples (EXMC 19, 28, 20, 29, 39, 31, and 33) were BPSV-VEGF negative. All others have positive signals for the poxvirus VEGF-E homologs (SI Fig. 2, middle panel). For the Non-matched control samples, only one sample (NC3) was found to be positive for BPSV-VEGF, and none of the others were positive for ORFV-VEGF-E or PCPVVEGF-E (SI Fig. 2, right panel). All samples, including the EXTN, EXMC, and NC were positive for the presence of human VEGFA transcripts as would be expected (SI Fig. 2). Therefore, our results indicate that more than $80 \%$ of the tumor samples are positive for VEGF-E as compared to approximately $60 \%$ for the matched controls. Importantly, only $10 \%$ of the non-matched tissues were positive for the VEGF-E.

\section{Parapoxvirus-encoded VEGF-E can modulate metabolic pathways}

One hallmark of all tumors is altered metabolism with upregulated glucose uptake and anaerobic respiration (glycolysis), and subsequent entry into the TCA cycle which is required for production of the available ATP and $\mathrm{NADPH}^{45}$. The amino acid glutamine is an essential amino acid that links carbohydrate metabolism to nucleotide biosynthesis. Hyper-proliferating cancer cells requires a steady supply of nucleotides and require
10-100 times more glutamine compared to normal cells ${ }^{46}$. Similarly, higher levels of de novo fatty acid synthesis from acetyl CoA, and mitochondrial betaoxidation are also observed in the cancer cells compared to normal cells ${ }^{29,47}$.

We selected 11 genes linked to carbohydrate metabolism, 5 genes linked to amino acid metabolism, and 6 genes involved in fatty acid metabolism and examined their transcriptional expression in the tissue samples. These genes were previously reported to be altered when examined in several cancers ${ }^{48}$. Our results showed that the tumor samples were found to have higher expression of these genes compared to that of the matched controls whereas the non-matched controls showed minimal expression (Fig. 2A-D).

Tumor samples have been shown to have a variety of different microbiome signatures compared to their matched control and non-matched normal tissue controls ${ }^{11,36,37}$. To confirm the involvement of the Poxvirusencoded VEGF-E in altered metabolism, three VEGF-E (ORFV-VEGF, PCPV-VEGF, and BPSV-VEGF) were cloned in Lentiviral plasmids with GFP (pLVX AcGFP) (SI Fig. 3A). All constructs were confirmed by sequencing (data not shown). The expressions were further determined at the protein level by western blot to monitor the level of the GFP-fused protein using GFP specific antibodies (SI Fig. 3B). The viral VEGFs were transduced by lentiviral vectors into triple negative breast cancer cells (MDA-MB-231) and the normal breast cells (HMEC) in vitro. The results showed upregulation of the selected metabolic genes in both cell types (Fig. 3A, B). Importantly, the fold increase in the levels of expression of the metabolic genes in stably transfected HMEC was much higher than that of the transfected MDA-MB-231 cells as compared to the non-transfected cells (SI Table 5). MDAMB-231 is a TNBC cell line, which already has upregulated expression of these metabolic genes while the HMEC cells are normal breast epithelial cells ${ }^{49}$. Therefore, transfection of normal breast cells with viralencoded VEGF homologs have a more pronounced effect on normal breast HMEC cells compared to that of the MDA-MB-231 cells (Fig. 3 and SI Table 5).

\section{Viral-encoded VEGF homologs can induce proliferation of breast epithelial cells}

Alteration of cellular proliferation was investigated using Bromouridine (BrdU) staining. MDA-MB-231 and HMEC cells were transduced with Lentivurus containing the viral-encoded VEGF homologs and was treated with BrdU for $24 \mathrm{~h}$ then fixed and perforated and stained with anti-BrdU antibody tagged with PE fluorophore and analyzed to determine their degree of proliferation (Fig. 4A).Viral-encoded VEGF homologs expressing cells showed much higher mean fluorescent intensity (MFI) 


\section{A}

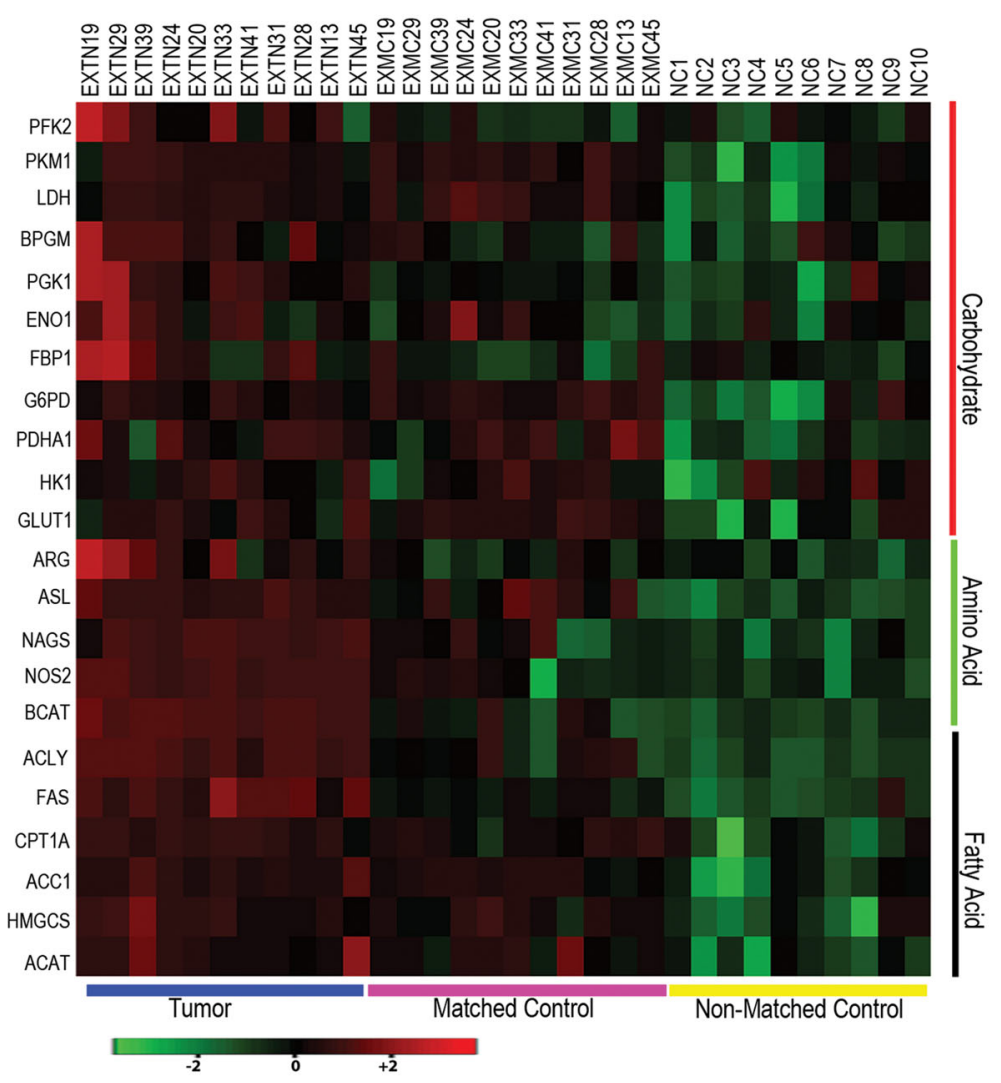

B

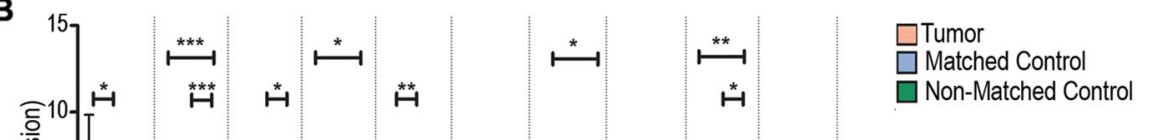

$\begin{array}{lllllllllll} & & & & \end{array}$

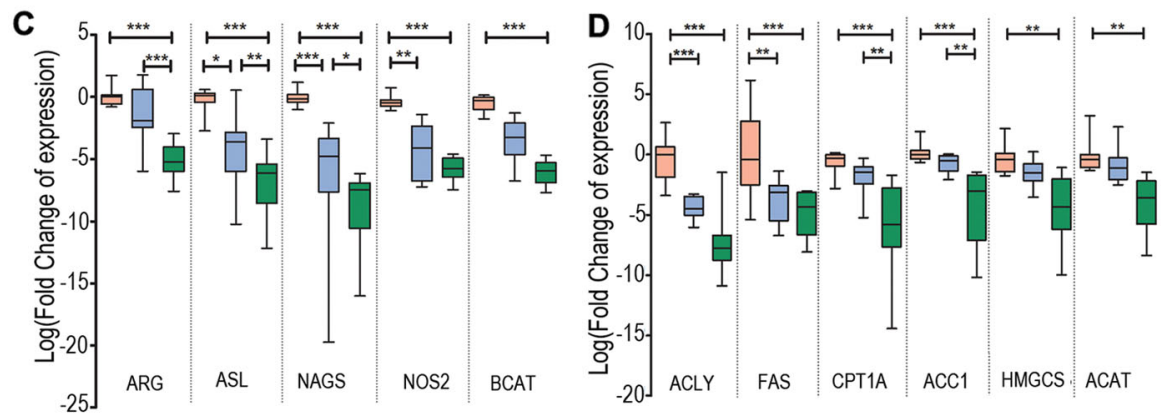

Fig. 2 (See legend on next page.) 
(see figure on previous page)

Fig. 2 Differential expression of the metabolic genes in tumor samples compared to the matched and non-matched control. The heat maps represent the logarithmic values of the expression levels of the different metabolic genes. A The fold change of the expression of each of the genes were represented in the heat maps. A set of 11 carbohydrate, 5 amino acid, and 6 lipid metabolism genes were examined. B-D The logarithmic values of the fold change of expression of all the genes of carbohydrate, amino acid, and fatty acid metabolism for each of the groups i.e., tumor, matched control, and non-matched control were represented as bars and are compared among themselves. The real-time PCR experiments were performed in duplicates, with two experimental repeats for each gene. ${ }^{*} P<0.05$; ${ }^{* *} P<0.005 ;{ }^{* * *} P<0.001$.
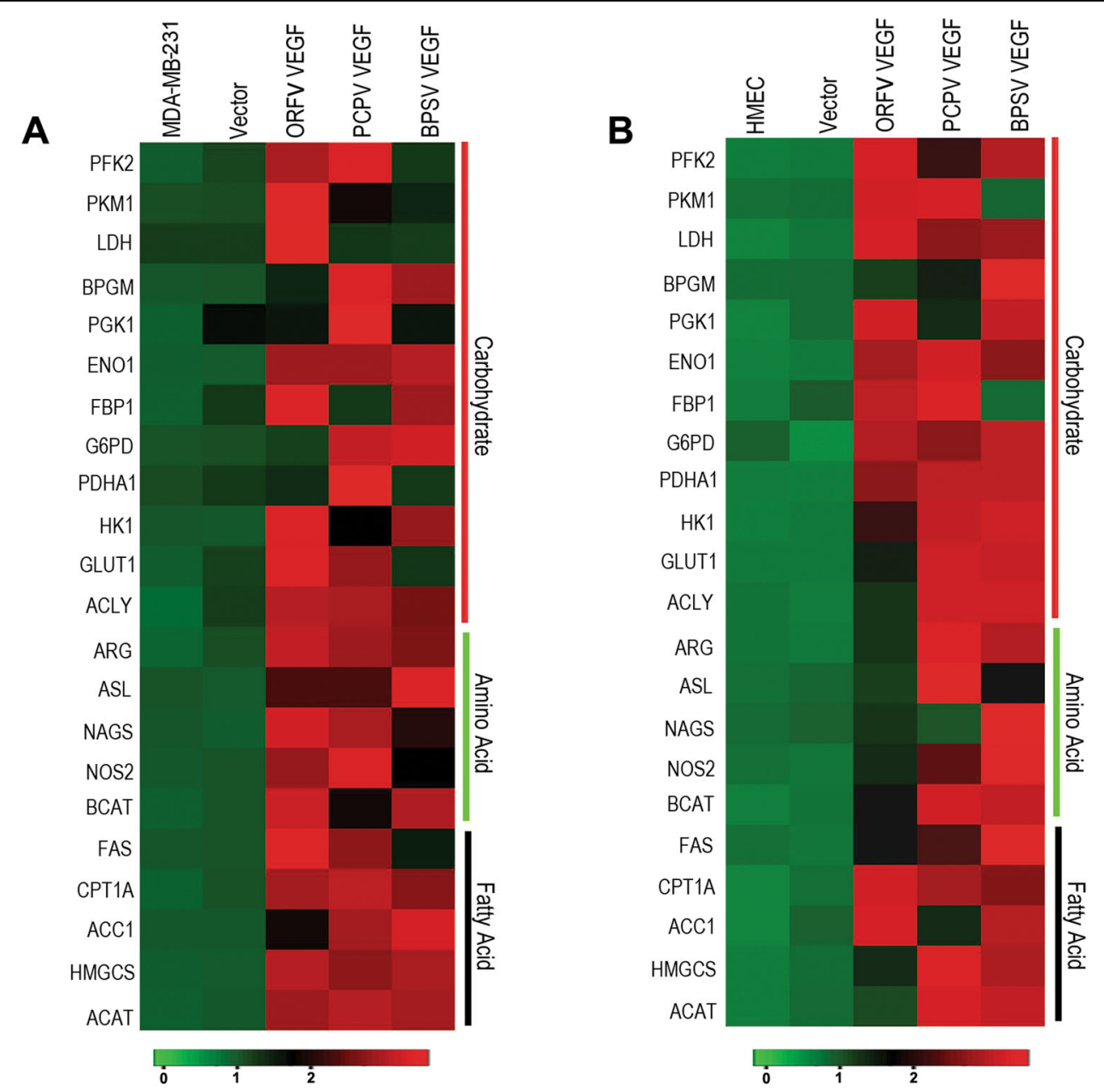

Fig. 3 Differential expression of the metabolic genes in VEGF transfected MDA-MB-231 and HMEC cells compared to the mock transfected cells. A MDA-MB-231 or B HMEC cells were transfected with empty vector, ORFV VEGF-E, PCPV VEGF-E or BPSV VEGF-E and relative expression of the metabolic genes were calculated using real-time PCR as compared to the non-transfected cells. The heat maps represent the logarithmic values of the fold change of the different metabolic genes compared to the non-transfected cells and normalized with 18S rRNA. A set of 11 carbohydrate, 5 amino acid, and 6 lipid metabolism genes were examined. The real-time PCR experiments were performed in duplicates, with two experimental repeats for each gene.

after compared to the empty vector transfected control (Fig. 4A). This demonstrated a higher proliferation rate compared to the mock transfected cells. In the case of HMEC cells, the change in mean fluorescence intensity (MFI) was, therefore, substantially greater when compared to the MDA-MB-231 cells (Fig. 4A). This variation may be because MDA-MB-231 cells are already transformed and therefore have a higher proliferation rate compared to the HMEC cells. Thus, the alteration in proliferation of HMEC was more significant when compared to the MDA-MB-231 cells which are already hyperproliferative due to their transformed state. 


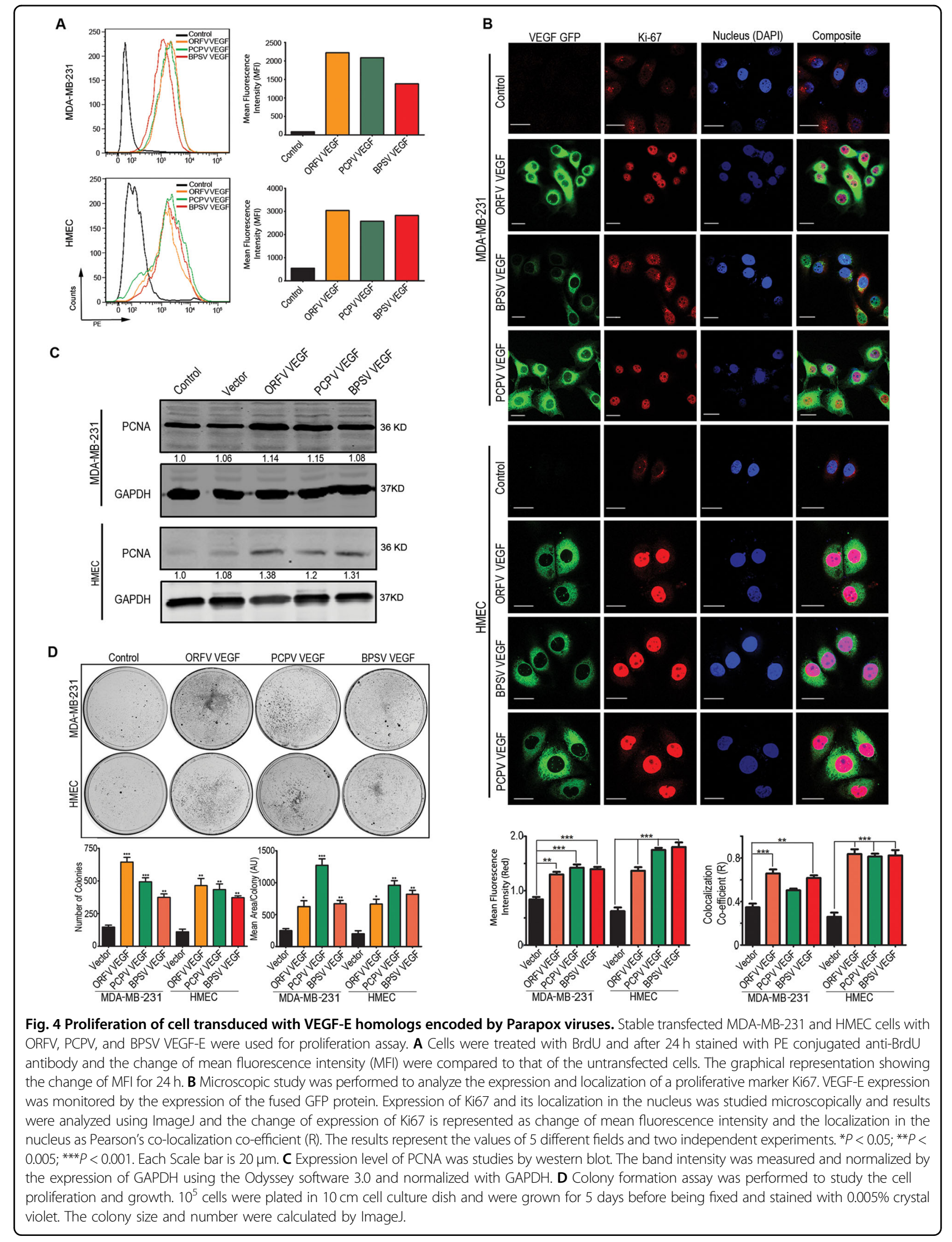




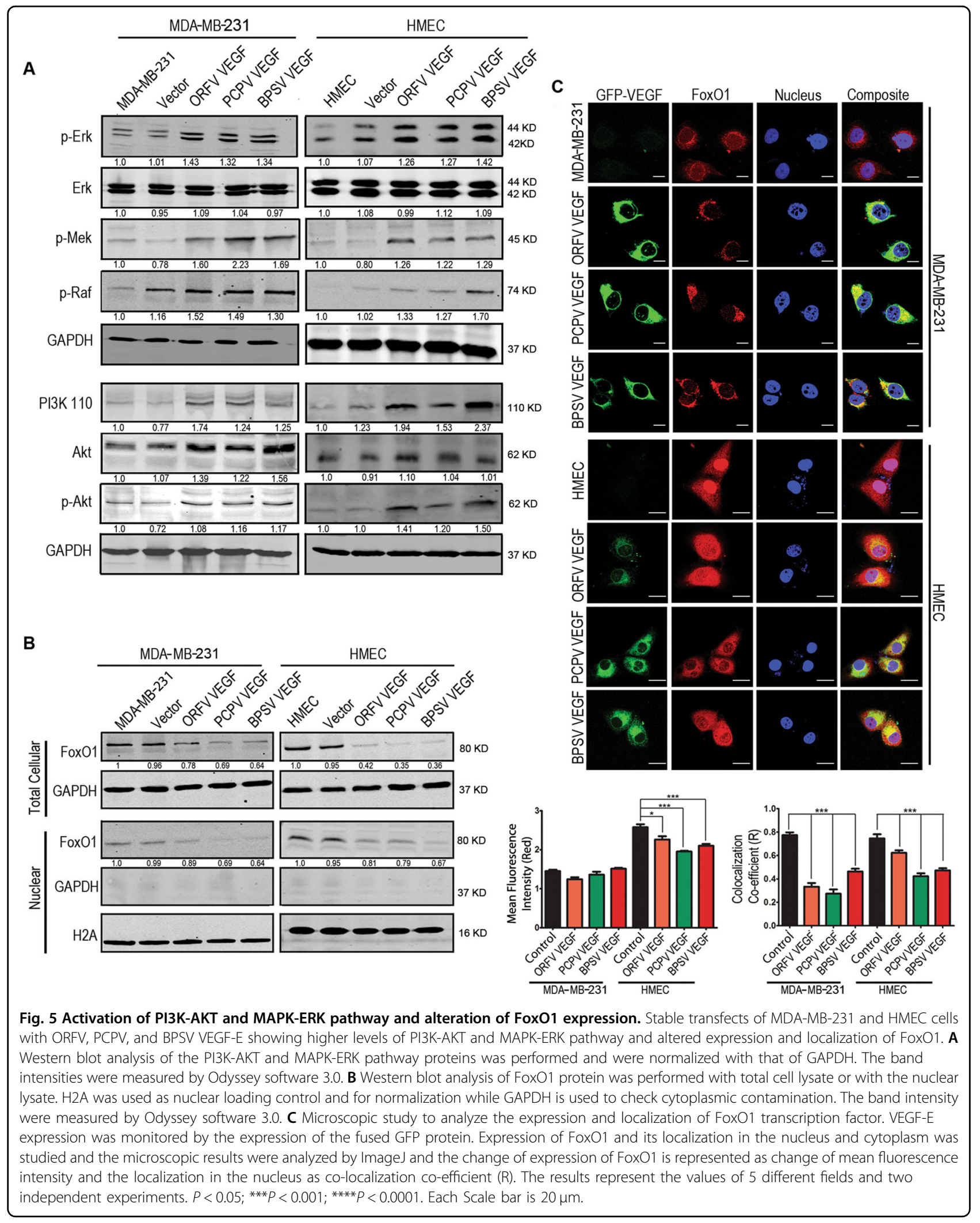


To provide additional evidence in support of these results, we used two different markers of proliferation, Ki67 and PCNA. Confocal microscopy of Ki67 showed higher expression, and its localization in the nucleus of cells expressing viral-encoded VEGF-E homologs compared to the non-transfected cells. The signals were plotted for mean fluorescence intensity and colocalization with the nucleus. Graphs showing the results are shown on right panels (Fig. 4B, top right panel). The expression levels of PCNA was also found to increase significantly in the transfected cells as demonstrated by western blot analysis for both cell lines stably expressing the VEGF homologs (Fig. 4C).

Colony formation assays were also performed to assess the proliferation rate and the ability of cells to form colonies. $10^{5}$ cells were plated, and colony formation was evaluated after 7 days. We observed a significant increase in the number and size of colonies in the cells stably expressing the VEGF homologs compared to the nontransfected cells indicating higher proliferations and increased oncogenic activities of these cells (Fig. 4D).

To further confirm that the secreted VEGF-E is responsible for the altered cellular growth, the VEGF-E or mock stable transfected cells (MDA-MB-231 and HMEC) were cultured and after $24 \mathrm{~h}$ the cell supernatant was collected and it was used at a ratio of 1:1 with fresh media to culture MDA-MB-231 and HMEC (without VEGF-E) and the proliferation was measured by MTT assay (SI Fig. $4 \mathrm{~A}$ ) and the data clearly shows that there is $1.3-1.8$ fold more cellular proliferation in presence of the viral VEGFE containing media compared to mock for MDA-MB-231 and 1.2-1.7 fold compared to mock for the HMEC cells suggesting the secreted VEGF-E is responsible for the cellular proliferation.

To further confirm that the secreted VEGF-E is responsible for the proliferation, the VEGF-E or mock stable transfected cells (MDA-MB-231 and HMEC) were cultured and after $24 \mathrm{~h}$ and the cell supernatant was collected and treated with protein A/G tagged anti-GFP neutralizing antibody for $4 \mathrm{~h}$ and centrifuged to precipitate the GFP fused viral VEGF-E protein from the supernatant and is used to treat MDA-MB231 and HMEC cells. The proliferation was measured by MTT assay (SI Fig. 4B). No significant change in proliferation observed between the cells treated with mock transfected media and viral VEGF transfected media. This finding clearly points out that the proliferative role of the viral VEGF-E.

\section{Viral-encoded VEGF-E can mediate PI3K-AKT pathway activation}

Previous reports have suggested that cellular VEGF, activated both the PI3K-AKT pathway and the ERKMAPK pathway ${ }^{50}$. The PI3K pathway includes AKT, which is known to enhance cell survival by stimulating cell proliferation and angiogenesis ${ }^{51}$. Similarly, human VEGF has also been shown to activate the MAPK-ERK pathway and promotes survival of cells ${ }^{52}$. Therefore, we examined the expression of a number of downstream proteins of these pathways. Our results showed that the pERK, p-MEK and p-RAF of the ERK-MAPK pathway, and p110 $\alpha$ and p-AKT of the PI3K-AKT pathway were found to be upregulated in both MDA-MB-231 and HMEC cells stably expressing the VEGF-E homologs from the Lentivirus when compared to the non-transfected cells (Fig. $5 \mathrm{~A}$ ). This suggests that the VEGF-E homologs can mediate the upregulation of these signaling pathways.

\section{Involvement of FoxO1 in metabolic gene expression}

The Fork head transcription factor (Fox or FHKR) is a group of four different transcription factors that are mainly involved in a number of pathophysiological processes like ageing, cancer, altered metabolism and cell death $^{53-55}$. FoxO1 is a member of the Fox family, which is mainly related to expression of different metabolic genes in response to insulin in patients suffering from diabetics ${ }^{56,57}$. However, recent reports also indicates that phosphorylation or methylation of FoxO1 sequesters it from the nucleus to the cytoplasm and can contribute to proliferation and altered metabolism in cancer $^{58-60}$. In our study, we were interested in examining the levels of FoxO1 and its localization in cells stably expressing the VEGF-E homologs. We found that both the total cellular and nuclear FoxO1 levels were reduced in cells expressing the VEGF-E homologs compared to the non-transfected or mock transfected cells (Fig. 5B). To further ascertain the localization of FoxO1, we performed fluorescence microscopic study which showed that expression of the VEGF-E homologs not only reduced the levels of FoxO1, but also led to translocation of FoxO1 from the nucleus to the cytoplasm as evident from the co-localization coefficient (Fig. 5C).

\section{VEGF-E homologs alters FoxO1 through PI3K-AKT pathway}

To further support the involvement of the PI3K pathway, we used LY294002, an inhibitor of the PI3K pathway ${ }^{61,62}$. LY294002 was found to inhibit the activity of PI3K as evident from the western blot analyses (Fig. 6A). PI3K activates AKT through phosphorylation at the serine 473 residue $^{63}$. LY294002 significantly decreased the pAKT levels suggesting inhibition of the pathway. We observed that the levels of FoxO1 increased in LY294002 treated cells compared to the cells expressing the VEGF-E homologs and suggest that inhibition of the PI3K pathway can led to accumulation of the total cellular FoxO1 (Fig. 6A).

As FoxO1 is related to expression of the metabolic genes, we investigated the mRNA levels of the different metabolic genes in LY294002 treated cells. LY294002 
A

VEGF pLVX pLVX ORFV ORFVBPSV BPSV PCPV PCPV $\frac{\text { MDA-MB } 231}{\mathrm{pLV} \text { pLVX ORFV ORFV BPSV BPSV PCPV PCPV }}$

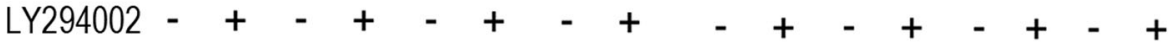

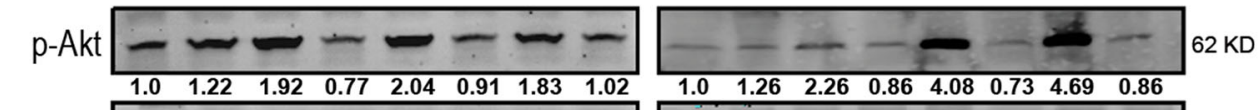

Akt
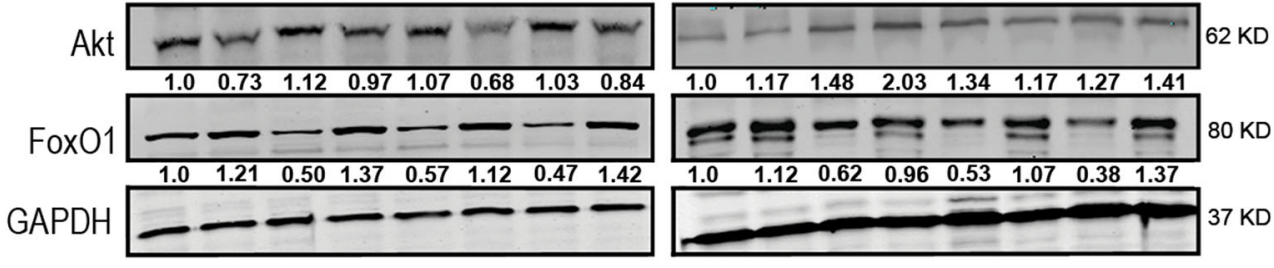

B

MDA-MB-231

VEGF $\mathrm{pLVX}$ pLVX ORFV ORFVBPSV BPSV PCPVPCPV

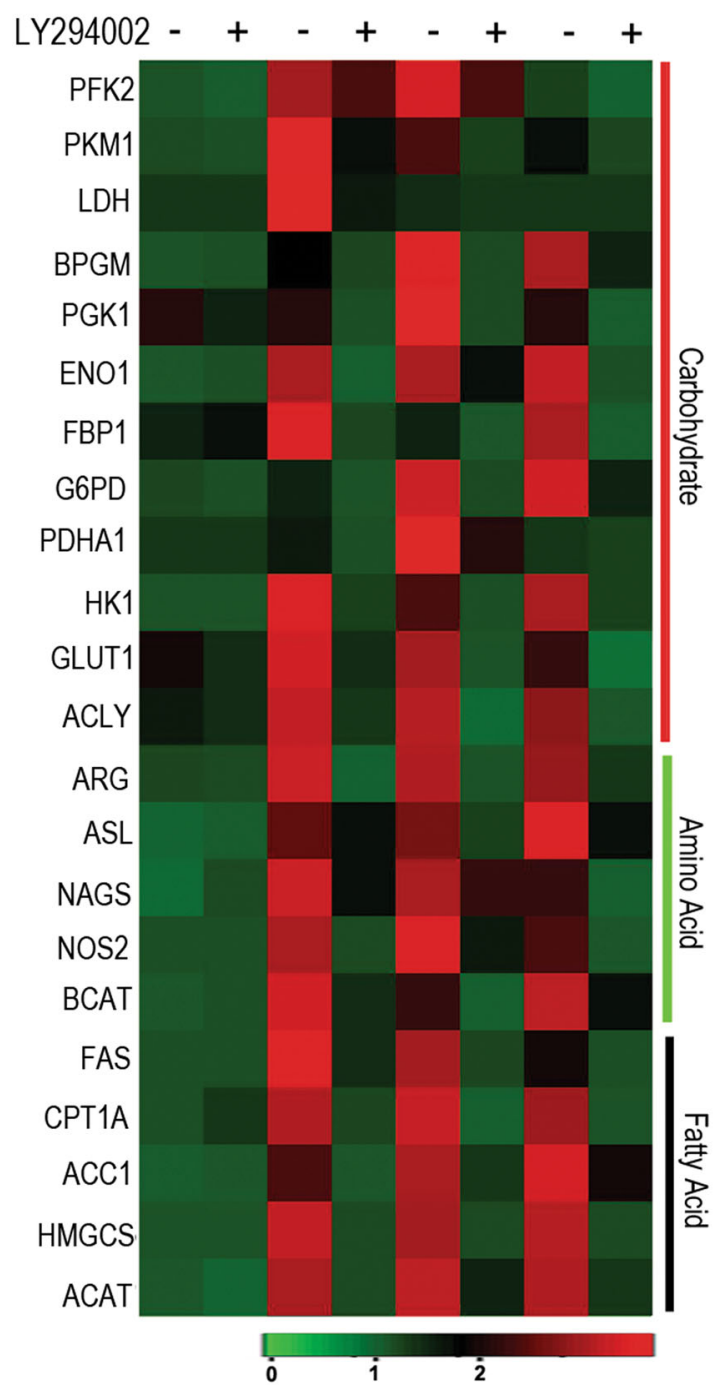

C

HMEC

VEGF pLVX PLVX ORFV ORFVBPSV BPSV PCPVPCPV

LY294002 - + - + - + - +

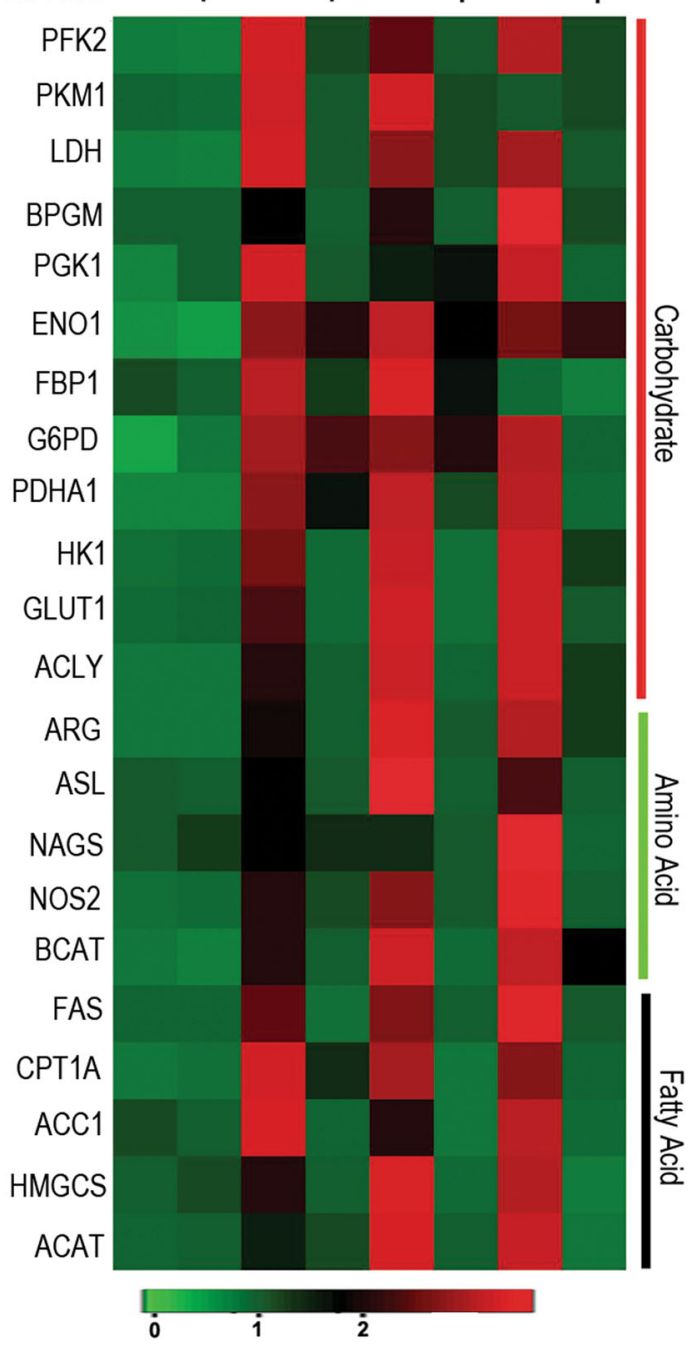

Fig. 6 (See legend on next page.) 
(see figure on previous page)

Fig. 6 Effect of LY294002 on the FoxO1 expression and on the expression of metabolic genes. Transfected or non-transfected cells were treated with LY294002 (50 $\mu$ M for MDA-MB-231 cells and $30 \mu \mathrm{M}$ for HMEC cells) and its downstream targets were monitored. A Western blot analysis was performed to analyze the effect of LY294002 on the levels of phosphorylation of AKT and FoxO1 and were compared with that of inhibitor untreated cells. The band intensity was measured by Odyssey software 3.0 and normalization was done with GAPDH. B, C Quantitative PCR was performed to check the expression pattern of the metabolic genes in presence or absence of LY294002 in mock or VEGF-E transfected MDA-MB-231 and HMEC cells. The heat maps represent the logarithmic values of the fold change of the different metabolic genes compared to the nontransfected cells.

treatment led to the downregulation of mRNA expression of a range of metabolic genes in cells expressing the VEGF-E homologs (Fig. 6B). These results clearly suggest that inhibition of the PI3K pathway in the cells expressing the VEGF-E homologs reversed the phenotype to that of the non-transfected cells and thus links the PI3K pathway to metabolic gene expression in the presence of the viralencoded VEGF-E homologs.

\section{Discussion}

The microbiota in combination with the host becomes a super-organism in which this symbiotic relationship is mutually beneficial ${ }^{64}$. However, a change in the host microenvironment promotes implantation and proliferation of dysbiotic microorganisms that may contribute to a range of different pathologies. These dysbiotic microorganisms has been linked to, and is being regarded as causative agents of a number of different pathophysiological conditions, including diabetes, cancer, atherosclerosis, and cardiac disorders ${ }^{37,65-67}$. The dysbiotic microbiota can also directly interact with cellular signaling pathways, or they can be activated by metabolites secreted by the associated microorganisms to initiate and maintain the pathogenic phenotype ${ }^{68}$. Dysbiosis in the gut microbiome has been linked to chronic inflammation that can result in cancers of the gastrointestinal track ${ }^{69}$. Moreover, there are also reports about abnormal gut microbiome, which contributes to the onset of breast cancer $^{70,71}$. However, there are no clear reports about the dysbiotic breast microbiome and its relationship to cancer progression.

Cancer cells are highly proliferating and, therefore, require a continuous supply of energy for their survival. To meet this high-energy demand, cancer cells have developed a different metabolic strategy compared to the normal cells ${ }^{72}$. TNBC are aggressive forms of breast cancer and due to the lack of the HER, ER, and PR receptors, most of the available hormonal therapies are thus ineffective.

There are few previous reports about dysbiotic microbiomes and their association with breast cancer $^{73}$. Previous report from our lab was one of the pioneering study on TNBC that extensively identified different dysbiotic microbiomes ${ }^{11}$. In the present study, we have screened a new cohort of 11 TNBC samples and its corresponding matched controls and 10 non-matched controls using the previous protocol ${ }^{43}$. We found that dsybiotic Parapoxvirus signatures are the highest intensity of resident microbial genome signatures that are predominately found in the tumor samples compared to the matched control and almost absent in the non-matched control, which were validated by PCR using specific primers for the Parapoxvirus families. The abundance of Parapoxvirus genome signatures in TNBC samples is congruent with our previous study, which was based on a larger cohort of 100 samples $^{11}$. We were also interested in investigating the status of metabolic genes in these samples and observed a dysregulated expression of some key glycolytic, fatty acid, and amino acid metabolic genes compared to the matched and non-matched controls. This suggests the role of the dysbiotic virome in regulation of the metabolic activities of breast cancer cells.

Some genes encoded by Parapoxviruses have sequence homology to that of their human counterpart and one of the most extensively studied encoded gene is the VEGF homolog (VEGF-E). Previous reports suggested that these VEGF homologs may bind to human VEGFR2 and trigger downstream signaling ${ }^{74}$. Therefore, we were interested in specifically studying the response of the TNBC cells to VEGF-E homolog expression. We were also curious to see the effect of these VEGF-E homologs on normal cells. We transduced MDA-MB-231 (TNBC cell line) and HMEC (normal breast epithelial cells) with the three different homologs of the Parapoxvirus-encoded VEGF-E and observed that the VEGF-E transduced cells showed higher growth rate and cell proliferation compared to the cells that do not express the homologs. Moreover we also found that the viral VEGF-E are secreted (SI Fig. 3C) and these secreted VEGF-E has the potential to induce a proliferative phenotype in the non-transfected cells (SI Fig. 4). Notably, VEGF-E transfected cells were also altered in their expression of metabolic genes and was quite comparable to that of the tumor samples examined. The effect of VEGF-E homologs on primary HMEC cells was also more prominent compared to that of the MDAMB-231 cells. This is probably due to the fact that MDAMB-231 cells is an already transformed cancer cell line with higher proliferation rates, with altered metabolism 


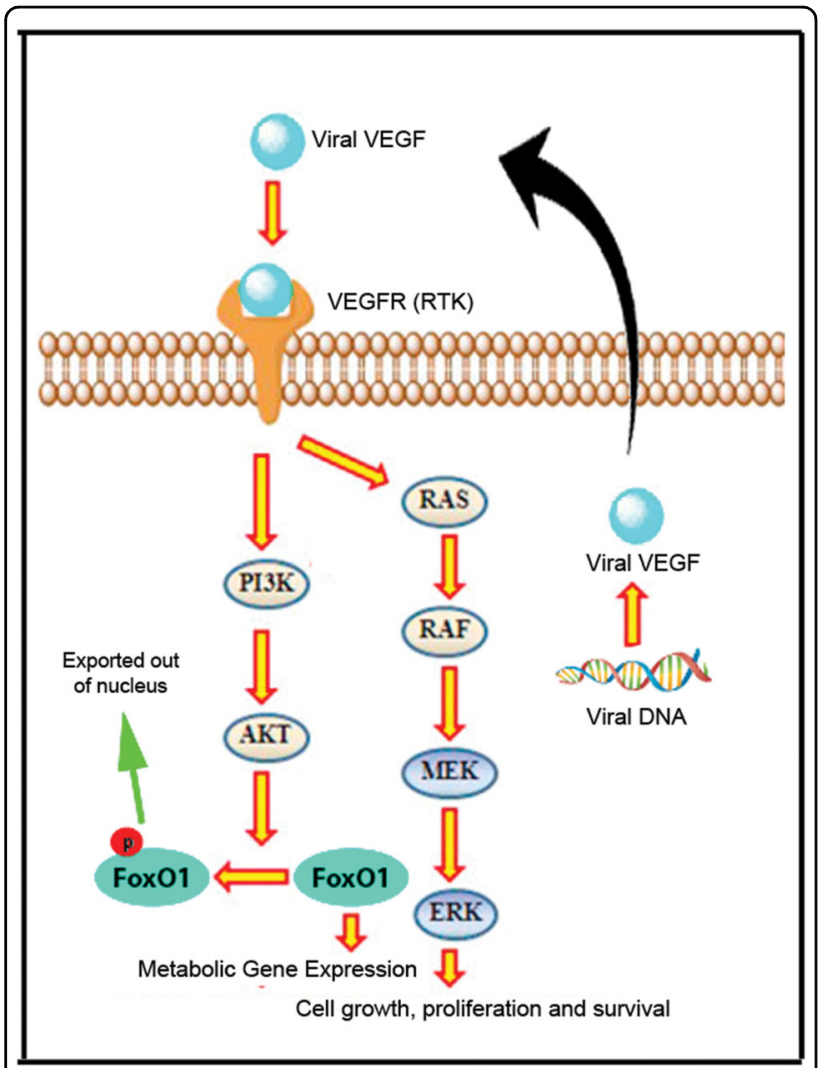

Fig. 7 Schematic representation showing VEGF-E modulating of cell proliferation and metabolism. Parapoxvirus, one of the dsybiotic microbiome in the breast cancer cells, encodes viral VEGF-E that acts through the VEGFR and triggers PI3K-AKT and MAPK-ERK pathway. Activated Erk and AKT pathway promotes cell growth, proliferation, and survival. In addition, activated AKT phosphorylates FoxO1 and shuttles its out of the nucleus where it is subsequently degraded. FoxO1 is regarded as tumor suppressor and is linked to expression of different metabolic genes. VEGF-E induced quenching and degradation of FoxO1 facilitates cellular proliferation with altered metabolism.

compared to the normal HMEC cells. Therefore, we would expect that the effect of the VEGF-E homologs would be more significant in the normal HMEC cells. There are previous reports that viral VEGF-E can stimulate cellular VEGF-A production ${ }^{43}$. To determine if the viral VEGF-E is the main driving factor for the enhanced proliferation, we have knocked down the cellular VEGF-A by shRNA and transfected those cells with the viral VEGF-Es and compared the cellular proliferation with the un-transfected cells (SI Fig. 5). Our data clearly showed that the secreted VEGF-E can induce a proliferative potential to non-transfected cells independent of the cellular VEGF-A.

As human VEGFA can activate the PI3K pathway ${ }^{75}$, we were interested in seeing whether the VEGF-E homologs had a similar effect. We found the VEGF-E homologs triggered the PI3K-AKT pathway and altered expression of FoxO1 transcription factor. FoxO1 is a critical transcription factor that not only controls cellular proliferation but is also responsible for regulating the transcription of a number of metabolic genes ${ }^{76}$, maintains homeostasis between metabolic pathways, and is also functionally noted as a tumor suppressor ${ }^{77}$. FoxO1 promotes gluconeogenesis, which typically shuttles TCA intermediates to form glucose ${ }^{78,79}$. Cancer cells, however, follow the opposite path and will avoid oxidative phosphorylation $^{18,80}$. During uncontrolled proliferation FoxO1 is shuttled back to the cytoplasm where it is degraded ${ }^{60}$. We found that the VEGF-E homologs exclude FoxO1 from the nucleus and sequestered it to the cytoplasm. Therefore this would promote the glycolytic pathway activity by downregulating gluconeogenesis, which in turn promotes cell proliferation.

Inhibition of the PI3K pathway counteracts the effect of the VEGF-E homologs through induction of FoxO1 levels inside the nucleus. This restored the metabolic gene expression levels similar to the cells which do not express the VEGF-E homologs. These results strongly suggested that the Parapoxvirus-encoded VEGF-E acts through the VEGFR2, thus upregulating the VEGFR2 expression in both MDA-MB-231 and HMEC cells (SI Fig. 6) leading to the upregulation of both the PI3K-AKT and ERK-MAPK pathways through sequestration of the FoxO1 transcription factor and altered the metabolic status of the cells (Fig. 7).

Our study provides some initial insights into the action of secretory factors (VEGF-E homologs) encoded by agents associated with a dysbiotic microbiome, which affects proliferation and altered metabolism in tumor cells. Our results also suggest that these VEGF homologs can also induce proliferation, and an altered metabolic phenotype in the normal cells as evident from our HMEC results. Here, we selected one of a plethora of factors from the dysbiotic microbiome in TNBC and dissected its potential role in cancer progression. These in vitro studies highlight the potential strategies utilized by microbial agents to survive and provide mutual benefits for host cells. However, in an in vivo microenvironment there are likely to be other factors produced by other agents in the microbiota that may also play a crucial role in disease pathogenesis. We also should stress that these findings do not directly demonstrate that these parapox viruses are directly responsible for TNBC but the DNA elements from these agents encodes proteins that contributes to the proliferative phenotype of the TNBC. Clearly, additional studies are necessary to further examine the activities of these other agents and their contributions to the disease.

\section{Acknowledgements}

The study was supported by Avon Foundation Grant no. Avon-02-2012-053 (to E.S.R.), and from the Abramson Cancer Center Director's fund. We also extend our gratitude to Xiang Lin and Dr. Zhi Wei from Department of Computer 
Science, New Jersey Institute of Technology, Newark, NJ, USA for their assistance on the analysis of the Pathochip results.

\section{Conflict of interest}

The authors declare that they have no conflict of interest.

\section{Publisher's note}

Springer Nature remains neutral with regard to jurisdictional claims in published maps and institutional affiliations.

Supplementary Information accompanies this paper at (https://doi.org/ 10.1038/s41419-020-03203-4).

Received: 14 April 2020 Revised: 2 November 2020 Accepted: 3 November 2020

Published online: 20 November 2020

\section{References}

1. Dai, $X$. et al. Breast cancer intrinsic subtype classification, clinical use and future trends. Am. J. Cancer Res. 5, 2929-2943 (2015).

2. Ovcaricek, T., Frkovic, S. G., Matos, E., Mozina, B. \& Borstnar, S. Triple negative breast cancer - prognostic factors and survival. Radio. Oncol. 45, 46-52 (2011).

3. Rea, D. et al. Microbiota effects on cancer: from risks to therapies. Oncotarget $\mathbf{9}$, 17915-17927 (2018).

4. Garrett, W. S. Cancer and the microbiota. Science 348, 80-86 (2015)

5. Atsumi, T. et al. Inflammation amplifier, a new paradigm in cancer biology. Cancer Res. 74, 8-14 (2014).

6. Francescone, R., Hou, V. \& Grivennikov, S. I. Microbiome, inflammation, and cancer. Cancer J. 20, 181-189 (2014).

7. Fulbright, L. E., Ellermann, M. \& Arthur, J. C. The microbiome and the hallmarks of cancer. PLoS Pathog. 13, e1006480 (2017).

8. Sender, R., Fuchs, S. \& Milo, R. Revised estimates for the number of human and bacteria cells in the body. PLOS Biol. 14, e1002533 (2016).

9. Littman, D. R. \& Pamer, E. G. Role of the commensal microbiota in normal and pathogenic host immune responses. Cell Host Microbe 10, 311-323 (2011).

10. DeGruttola, A. K., Low, D., Mizoguchi, A. \& Mizoguchi, E. Current Understanding of Dysbiosis in Disease in Human and Animal Models. Inflamm. Bowel Dis. 22, 1137-1150 (2016).

11. Banerjee, S. et al. Distinct microbiological signatures associated with triple negative breast cancer. Sci. Rep. 5, 15162 (2015)

12. Meyer, M. et al. A novel vascular endothelial growth factor encoded by Orf virus, VEGF-E, mediates angiogenesis via signalling through VEGFR-2 (KDR) but not VEGFR-1 (Flt-1) receptor tyrosine kinases. EMBO J. 18, 363-374 (1999).

13. Savory, L. J., Stacker, S. A., Fleming, S. B., Niven, B. E. \& Mercer, A. A. Viral vascular endothelial growth factor plays a critical role in orf virus infection. J. Virol. 74, 10699-10706 (2000)

14. Lyttle, D. J., Fraser, K. M., Fleming, S. B., Mercer, A. A. \& Robinson, A. J. Homologs of vascular endothelial growth factor are encoded by the poxvirus orf virus. J. Virol. 68, 84-92 (1994).

15. Cebe-Suarez, S., Zehnder-Fjallman, A. \& Ballmer-Hofer, K. The role of VEGF receptors in angiogenesis; complex partnerships. Cell Mol. Life Sci. 63, 601-615 (2006).

16. Wise, L. M. et al. Vascular endothelial growth factor (VEGF)-like protein from orf virus NZ2 binds to VEGFR2 and neuropilin-1. Proc. Natl Acad. Sci. USA 96, 3071-3076 (1999).

17. Samudio, I., Fiegl, M. \& Andreeff, M. Mitochondrial uncoupling and the Warburg effect: molecular basis for the reprogramming of cancer cell metabolism. Cancer Res 69, 2163-2166 (2009).

18. Balsa-Martinez, E. \& Puigserver, P. Cancer Cells Hijack Gluconeogenic Enzymes to Fuel Cell Growth. Mol. Cell 60, 509-511 (2015).

19. Altman, B. J., Stine, Z. E. \& Dang, C. V. From Krebs to clinic: glutamine metabolism to cancer therapy. Nat. Rev. Cancer 16, 619-634 (2016).

20. Aiderus, A., Black, M. A. \& Dunbier, A. K. Fatty acid oxidation is associated with proliferation and prognosis in breast and other cancers. BMC Cancer 18, 805 (2018).

21. Avtandilyan, N., Javrushyan, H., Petrosyan, G. \& Trchounian, A. The involvement of arginase and nitric oxide synthase in breast cancer development: arginase and NO synthase as therapeutic targets in cancer. Biomed. Res. Int. 2018 8696923 (2018)

22. Huang, H. L. et al. Argininosuccinate lyase is a potential therapeutic target in breast cancer. Oncol. Rep. 34, 3131-3139 (2015).

23. Suh, E. H. et al. In vivo assessment of increased oxidation of branched-chain amino acids in glioblastoma. Sci. Rep. 9, 340 (2019).

24. Flavin, R., Peluso, S., Nguyen, P. L. \& Loda, M. Fatty acid synthase as a potential therapeutic target in cancer. Future Oncol. 6, 551-562 (2010).

25. Pucci, $\mathrm{S}$. et al. Carnitine palmitoyl transferase-1A (CPT1A): a new tumor specific target in human breast cancer. Oncotarget 7, 19982-19996 (2016).

26. Brusselmans, K. De Schrijver, E., Verhoeven, G. \& Swinnen, J. V. RNA interference-mediated silencing of the acetyl-CoA-carboxylase-alpha gene induces growth inhibition and apoptosis of prostate cancer cells. Cancer Res. 65, 6719-6725 (2005)

27. Chen, S. W. et al. HMGCS2 enhances invasion and metastasis via direct interaction with PPARalpha to activate Src signaling in colorectal cancer and oral cancer. Oncotarget 8, 22460-22476 (2017).

28. Antalis, C. J. et al. High ACAT1 expression in estrogen receptor negative basallike breast cancer cells is associated with LDL-induced proliferation. Breast Cancer Res. Treat. 122, 661-670 (2010).

29. Currie, E., Schulze, A., Zechner, R., Walther, T. C. \& Farese, R. V. Jr Cellular fatty acid metabolism and cancer. Cell Metab. 18, 153-161 (2013).

30. de Ruijter, T. C., Veeck, J., de Hoon, J. P., van Engeland, M. \& Tjan-Heijnen, V. C. Characteristics of triple-negative breast cancer. J. Cancer Res. Clin. Oncol. 137 183-192 (2011).

31. Butti, R. et al. Receptor tyrosine kinases (RTKs) in breast cancer: signaling, therapeutic implications and challenges. Mol. Cancer 17, 34 (2018).

32. Lichtenberger, B. M. et al. Autocrine VEGF signaling synergizes with EGFR in tumor cells to promote epithelial cancer development. Cell 140, 268-279 (2010).

33. Domigan, C. K. et al. Autocrine VEGF maintains endothelial survival through regulation of metabolism and autophagy. J. Cell Sci. 128, 2236-2248 (2015).

34. Papa, S., Choy, P. M. \& Bubici, C. The ERK and JNK pathways in the regulation of metabolic reprogramming. Oncogene 38, 2223-2240 (2019).

35. Yu J J S. \& Cui, W. Proliferation, sunvival and metabolism: the role of PI3K/AKT/ mTOR signalling in pluripotency and cell fate determination. Development 143, 3050-3060 (2016).

36. Banerjee, S. et al. Microbiome signatures in prostate cancer. Carcinogenesis 40, 749-764 (2019).

37. Banerjee, S. et al. Distinct microbial signatures associated with different breast cancer types. Front Microbiol 9, 951 (2018).

38. Neve, R. M. et al. A collection of breast cancer cell lines for the study of functionally distinct cancer subtypes. Cancer Cell 10, 515-527 (2006).

39. Ueda, N., Wise, L. M., Stacker, S. A., Fleming, S. B. \& Mercer, A. A. Pseudocowpox virus encodes a homolog of vascular endothelial growth factor. Virology $\mathbf{3 0 5}$ 298-309 (2003).

40. Rothaeusler, K. \& Baumgarth, N. Assessment of cell proliferation by 5bromodeoxyuridine (BrdU) labeling for multicolor flow cytometry. Curr. Protoc. Cytom. Chapter 7, Unit7.31 (2007)

41. Schreiber, E. et al. Astrocytes and glioblastoma cells express novel octamerDNA binding proteins distinct from the ubiquitous Oct-1 and B cell type Oct-2 proteins. Nucleic Acids Res. 18, 5495-5503 (1990).

42. Babicki, S. et al. Heatmapper: web-enabled heat mapping for all. Nucleic Acids Res. 44, W147-W153 (2016).

43. Banerjee, S. et al. Identification of fungal pathogens in a patient with acute myelogenic leukemia using a pathogen detection array technology. Cancer Biol. Ther. 17, 339-345 (2016).

44. Wise, L. M. et al. The vascular endothelial growth factor (VEGF)-E encoded by orf virus regulates keratinocyte proliferation and migration and promotes epidermal regeneration. Cell Microbiol. 14, 1376-1390 (2012).

45. Pavlova, N. N. \& Thompson, C. B. The emerging hallmarks of cancer metabolism. Cell Metab. 23, 27-47 (2016).

46. DeBerardinis, R. J. \& Cheng, T. Q's next: the diverse functions of glutamine in metabolism, cell biology and cancer. Oncogene 29, 313-324 (2010).

47. Ma, Y. et al. Fatty acid oxidation: an emerging facet of metabolic transformation in cancer. Cancer Lett. 435, 92-100 (2018).

48. Oermann, E. K., Wu, J., Guan, K. L. \& Xiong, Y. Alterations of metabolic genes and metabolites in cancer. Semin Cell Dev. Biol. 23, 370-380 (2012).

49. Jia, D. et al. Elucidating cancer metabolic plasticity by coupling gene regulation with metabolic pathways. Proc. Natl Acad. Sci. USA 116 3909-3918 (2019). 
50. Meadows, K. N., Bryant, P. \& Pumiglia, K. Vascular endothelial growth factor induction of the angiogenic phenotype requires Ras activation. J. Biol. Chem. 276, 49289-49298 (2001).

51. Vivanco, I. \& Sawyers, C. L. The phosphatidylinositol 3-Kinase AKT pathway in human cancer. Nat. Rev. Cancer 2, 489-501 (2002).

52. Dhillon, A. S., Hagan, S., Rath, O. \& Kolch, W. MAP kinase signalling pathways in cancer. Oncogene 26, 3279-3290 (2007).

53. Fu, Z. \& Tindall, D. J. FOXOs, cancer and regulation of apoptosis. Oncogene 27, 2312-2319 (2008)

54. Martins, R., Lithgow, G. J. \& Link, W. Long live FOXO: unraveling the role of FOXO proteins in aging and longevity. Aging Cell 15, 196-207 (2016).

55. Accili, D. \& Arden, K. C. FoxOs at the crossroads of cellular metabolism, differentiation, and transformation. Cell 117, 421-426 (2004).

56. I, O. S. et al. FoxO1 integrates direct and indirect effects of insulin on hepatic glucose production and glucose utilization. Nat. Commun. 6, 7079 (2015).

57. Matsumoto, M., Han, S., Kitamura, T. \& Accili, D. Dual role of transcription factor FoxO1 in controlling hepatic insulin sensitivity and lipid metabolism. J. Clin. Invest 116, 2464-2472 (2006)

58. Link, W. \& Fernandez-Marcos, P. J. FOXO transcription factors at the interface of metabolism and cancer. Int J. Cancer 141, 2379-2391 (2017).

59. Lien, E. C., Lyssiotis, C. A. \& Cantley, L. C. Metabolic reprogramming by the PI3K-Akt-mTOR pathway in cancer. Recent Results Cancer Res. 207, 39-72 (2016).

60. Chae, Y. C. et al. FOXO1 degradation via G9a-mediated methylation promotes cell proliferation in colon cancer. Nucleic Acids Res. 47, 1692-1705 (2019).

61. Avni, D., Glucksam, Y. \& Zor, T. The phosphatidylinositol 3-kinase (PI3K) inhibitor LY294002 modulates cytokine expression in macrophages via p50 nuclear factor kappaB inhibition, in a PI3K-independent mechanism. Biochem. Pharm. 83, 106-114 (2012).

62. Jiang, H., Fan, D., Zhou, G., Li, X. \& Deng, H. Phosphatidylinositol 3-kinase inhibitor(LY294002) induces apoptosis of human nasopharyngeal carcinoma in vitro and in vivo. J. Exp. Clin. Cancer Res. 29, 34 (2010).

63. Paplomata, E. \& O'Regan, R. The PI3K AKT/mTOR pathway in breast cancer: targets, trials and biomarkers. Ther. Adv. Med Oncol. 6, 154-166 (2014).

64. Baquero, F. \& Nombela, C. The microbiome as a human organ. Clin. Microbiol Infect. 18(Suppl 4), 2-4 (2012).
65. Upadhyaya, S. \& Banerjee, G. Type 2 diabetes and gut microbiome: at the intersection of known and unknown. Gut Microbes 6, 85-92 (2015).

66. Bhatt, A. P., Redinbo, M. R. \& Bultman, S. J. The role of the microbiome in cancer development and therapy. CA Cancer J. Clin. 67, 326-344 (2017).

67. Kelly, J. R., Minuto, C., Cryan, J. F., Clarke, G. \& Dinan, T. G. Cross talk: the microbiota and neurodevelopmental disorders. Front Neurosci. 11, 490 (2017).

68. Grigg, J. B. \& Sonnenberg, G. F. Host-microbiota interactions shape local and systemic inflammatory diseases. J. Immunol. 198, 564-571 (2017).

69. Greenblum, S., Turnbaugh, P. J. \& Borenstein, E. Metagenomic systems biology of the human gut microbiome reveals topological shifts associated with obesity and inflammatory bowel disease. Proc. Natl Acad. Sci. USA 109 594-599 (2012).

70. Fernandez, M. F. et al. Breast cancer and its relationship with the microbiota. Int J Environ Res. Public Health 15, 1747 (2018).

71. Plaza-Diaz, J. et al. Association of breast and gut microbiota dysbiosis and the risk of breast cancer: a case-control clinical study. BMC Cancer 19, 495 (2019).

72. DeBerardinis, R. J. \& Chandel, N. S. Fundamentals of cancer metabolism. Sci. Adv. 2, e1600200 (2016).

73. Chen, J. et al. The microbiome and breast cancer: a review. Breast Cancer Res. Treat. 178, 493-496 (2019).

74. Shibuya, M. Vascular endothelial growth factor (VEGF) and Its receptor (VEGFR) Signaling in angiogenesis: a crucial target for anti- and pro-angiogenic therapies. Genes Cancer 2, 1097-1105 (2011).

75. Ruan, G. X. \& Kazlauskas, A. VEGF-A engages at least three tyrosine kinases to activate PI3KJAkt. Cell Cycle 11, 2047-2048 (2012).

76. Kousteni, S. FoxO1, the transcriptional chief of staff of energy metabolism. Bone 50, 437-443 (2012).

77. Xie, L. et al. FOXO1 is a tumor suppressor in classical Hodgkin lymphoma. Blood 119, 3503-3511 (2012).

78. Oh, K. J., Han, H. S., Kim, M. J. \& Koo, S. H. CREB and FoxO1: two transcription factors for the regulation of hepatic gluconeogenesis. BMB Rep. 46, 567-574 (2013).

79. Puigserver, P. et al. Insulin-regulated hepatic gluconeogenesis through FOXO1-PGC-1alpha interaction. Nature 423, 550-555 (2003).

80. Wang, Z. \& Dong, C. Gluconeogenesis in cancer: function and regulation of PEPCK, FBPase, and G6Pase. Trends Cancer 5, 30-45 (2019). 